\title{
Effectiveness of movement-based animal signals is a function of display structure and habitat characteristics: simulations of Australian dragons
}

\section{Xue Bian}

La Trobe University

Angela Pinilla

Monash University

Tom Chandler

Monash University

Richard Peters ( $\square$ richard.peters@latrobe.edu.au )

La Trobe University

\section{Research Article}

Keywords: animal communication, habitat structure, movement-based signal, visual saliency, signal evolution, 3D animation

Posted Date: December 15th, 2020

DOl: https://doi.org/10.21203/rs.3.rs-124579/v1

License: (9) This work is licensed under a Creative Commons Attribution 4.0 International License. Read Full License

Version of Record: A version of this preprint was published at Scientific Reports on March 18th, 2021. See the published version at https://doi.org/10.1038/s41598-021-85793-3. 


\section{Abstract}

Habitat-specific characteristics can affect signal transmission such that different habitats dictate the optimal signal. One way to examine how the environment influences signals is by comparing changes in signal efficacy in different habitats. Examinations of signal efficacy between different habitats has helped to explain signal divergence/convergence between populations and species utilising acoustic and colour signals. Although previous research has provided evidence for local adaptations and signal divergence in many species of lizards, comparative studies in movement-based signals are rare due to technical difficulties in quantifying movements in nature and ethical restrictions in translocating animals between habitats. We demonstrate herein that these issues can be addressed using 3D animations, and compared the relative performance of the displays of four Australian lizard species in the habitats of each species under varying environmental conditions. Our simulations show that habitats differentially affect signal performance, and an interaction between display and habitat structure. Interestingly, the signal adapted to the noisier environment did not show an advantage in signal efficacy, but the noisy habitat was detrimental to the performance of all displays. Our study is one of the first studies for movement-based signals that directly compares signal performance in multiple habitats, and our approach has laid the foundation for future investigations in motion ecology that have been intractable to conventional research methods.

\section{Introduction}

Animal signals evolve to be effective in the environment in which they are emitted [1-3], and therefore the diversity we see in animal communication systems is partially driven by the environment in which the animal lives [4-5]. An effective signal requires reliable detection by an intended receiver following transmission through ecologically complex environments [1]. It is evident that environmental structures and habitat-specific characteristics can affect signal transmission $[1,4,6-7]$, such that different habitats dictate the optimal signal for reliable detection [8]. Dynamically changing abiotic and biotic noise in the environment also interferes with effective communication between individuals, and animals are required to produce signals that could compete with these irrelevant sensory inputs [3,9-10]. Many animals modify their signalling behaviour in response to changing conditions [11-15], such that these moment-tomoment adjustments to signalling strategies represent a form of behavioural plasticity to maintain signal efficacy [16-17]. Clearly, habitat-specific transmission properties are important in determining the optimal structure of a signal, and it is therefore imperative that we quantify signal efficacy in the context of the relevant noise landscape to understand in more detail the evolution of animal communication strategies.

One way to examine how the environment influences signals is by comparing changes in signal efficacy in different habitats. Earlier research into quantifying acoustic characteristics of different habitat types provided the foundation for understanding diversity in the physical structure of avian songs $[6,18]$. Crosshabitat examination of signal efficacy between different habitats also helped to explain intraspecific divergence of calling behaviours in anuran species [19]. Similarly, by contrasting signals in different habitats, signal divergence was described for lizard populations utilising colour-based signals [20], as 
well as closely related insect species utilising seismic communication strategies [21]. Structural differences in movement-based signals have also been observed in many lizard species occupying both similar and distinctive habitats [17, 22-24]. Bloch and Irschick [25] found temporal differences in the display sequences of the green anole, Anolis carolinensis, suggesting population divergence due to population density and habitat use. Similarly, structural differences in the core display of the Jacky dragon, Amphibolurus muricatus, between three different populations might be a consequence of behavioural plasticity in response to variation in habitat structure [26]. Clearly, movement-based signals also show variation consistent with local adaptation explanations, but detailed cross-habitat comparisons of signal structure has rarely been demonstrated. Aside from the technical difficulties associated with quantifying the environmental conditions and animal displays [27], there are important legislative and ethical restrictions that prevent translocating animals between habitats that they do not naturally inhabit.

Plant movements are the primary source of motion noise that affects movement- based signal communication [14, 28-31]. However, the motion noise landscape is difficult to examine in a controlled, systematic way, as it differs vastly from one microhabitat to another as a result of the interaction between variable plant characteristics, habitat topography and microhabitat structure [32]. To our knowledge, the recent work by Ramos and Peters [33-34] represents the only attempts to consider the relative effectiveness of movement-based signals of lizards in multiple habitats. In the first of these studies, evidence is presented for local adaptation, showing that two sympatric lizard species who utilise different movements in their displays, nonetheless produced similar motion speeds [33]. In the second paper, the authors showed structural differences between allopatric populations of the same species [34]. In both studies, habitat structure was considered to be the guiding force leading to convergence or divergence in structure respectively. The approach taken in both studies, however, was to film displays in local environments, and then the movement of plants at a separate time, in a controlled manner, and to then contrast resultant motion speed data [35]. Although these are novel and informative insights, we have developed new techniques based around sophisticated three-dimensional (3D) animations that affords us the opportunity to consider movement-based signals embedded in noise [27].

The aim of the present study was to consider directly the relative effectiveness of the movement-based displays of multiple lizard species in multiple habitats using 3D animation. We reused the subject of earlier work [27, 36], the Jacky lizard, $A$. muricatus, and created three additional model lizards and their respective habitats: the long-nosed dragon, Gowidon longirostris, the Mallee dragon, Ctenophorus fordi, and the tawny dragon, $C$. decresii (Figure S1,S2). In addition to quite distinct signals, these four species also occupy different habitat types, from densely vegetated coastal heath environments to sparsely vegetated rocky outcrops and semi-arid Mallee woodland with spinifiex understories (Figure S2). The associated noise landscape in these habitats also varies as a result of vegetation density and plant species present. Signals adapted to a noisier environment tend to perform better when placed in a less noisy environment [33-34]. Therefore, we predicted that the signal of $A$. muricatus would perform better in the other three habitats as it occupies a densely vegetated and noisy environment. Similarly, we would also expect the habitat of $A$. muricatus to have the greatest influence on signal performance. The 
habitats of $G$. longirostris and $C$. decresii are very similar, so we predicted that these will influence signal performance of all species in a similar manner, and that their displays would perform equivalently. Ctenophorus fordi, on the other hand, would produce relatively weak signals as this species utilises the simplest motor pattern compared to the other three species.

Habitat structure is a crucial determinant of signal structure across species and modalities and here we extend this literature to include the movement-displays of lizards from different habitats. The diverse habitats are each inhabited by only one of the focal species, and experimental translocation of absent species would not be permissible. While we have simulated these circumstances, 3D animation technology is sufficiently advanced that these are excellent proxies for filming the same circumstances in nature (if it were possible). Our animations are detailed and founded on the natural systems they represent, while our analytical approach is quantitative but descriptive, and shows clearly that habitat structure is an important contributing factor for movement-based signalling systems.

\section{Methods}

\section{Study species}

Amphibolurus muricatus (snout-vent length, SVL, $121 \mathrm{~mm}$ ) is a well-known species that has featured in many physiology, ecology and visual communication studies [14, 37-40]. The display of $A$. muricatus begins with tail-flicking, followed by limb movements and a push-up, sometimes accompanied by throat extensions [41-42](Fig. 1a). There are two parts to the tail-flick sequence: an initial intermittent movement at the tip of the tail followed by a continuous and more rigorous movement of the whole tail. The tail-flicks serve as an introductory component to the rest of the display and are used to attract the attention of the intruder [31, 43]. Amphibolurus muricatus inhabits woodlands and coastal heath of southeast Australia and are often seen perched on fallen timber [44]. The simulated A. muricatus habitat was based on sites around the Croajingolong National Park, Victoria, where the habitat is densely vegetated with a complex understory of tall grasses and shrubs (Figure S3a).

In contrast to A. muricatus, the displays of Ctenophorus fordi, are much reduced in complexity, and almost exclusively comprised of head bobbing (Fig. 3c). Ctenophorus fordi are found in south-east Western Australia to western Victoria and New South Wales [44]. The species is smaller in size (SVL $58 \mathrm{~mm}$ ) and lack obvious sexual dimorphism [45-46]; although Garcia et al. [47] suggests they are sexually dimorphic in appearance in the ultraviolet region of the spectrum. Displays are performed by both sexes and triggered when another individual (even other species of lizards) appears in their field of vision [46]. The 3D model used herein was based on morphology and display footage taken from a population that inhabit dense clumps of spinifiex grass (Triodia sp.) and Mallee eucalyptus (Eucalyptus sp.) in the Murray-Sunset National Park, Victoria (Figure S3c).

Both $G$. longirostris and $C$. decresii are highly territorial species that inhabit semi-arid rocky outcrops with low vegetation density. Gowidon longirostris are relatively large lizards (SVL $114 \mathrm{~mm}$ ) mainly due to their exceedingly long tail compared to the other three species. The species is widely distributed in arid zones 
across western and central Australia [44]. The complex signalling sequence comprises limb waving and head bobbing, with push-ups followed by a forward body thrust/rise usually associated with tail lashing motions [48]. Throat and crest extensions are also common modifiers of the display (Fig. 1b). The model lizard used herein was based on morphology and display footage taken from a population located within and around Ormiston Gorge, within West-MacDonnell National Park, Western Australia. The habitat around Ormiston Gorge features large rocky outcrops with sparsely distributed vegetation including Eucalyptus trees, low shrubs (Acacias $s p$.) and a variety of native grasses and grass trees (Xanthorrhoea sp. Figure S3b).

Ctenophorus decresii are averaged sized lizards (SVL $82 \mathrm{~mm}$ ) found across eastern South Australia, including Kangaroo Island [49]. The species exhibits diverse colouration within and between populations [50] and is known to show individual variation in their agonistic displays [51]. Other than throat/crest extension, limb waving and head bobbing, $C$. decresii also displays a distinctive hind-leg push up with the tail coiled in a horizontal direction [49,52](Fig. 1d). The hind- leg push up is utilised by males to reflect endurance and aggressiveness during territorial interactions [51]. The model lizard used herein was based on morphology and display footages of $C$. decresii lizards inhabiting the rocky outcrops of the Flinders Ranges National Park, South Australia. The habitat is prone to fire, so we have incorporated charred fallen branches and sparse vegetation coverage, featuring mainly saltbush (Atriplex nummularia) and spinifex grass (Triodia sp.), as well as sparsely distributed Eucalyptus sp. and black oaks (Casurina sp.; Figure S3d).

\section{Creating and animating the habitats}

The microhabitats were modelled using Autodesk Maya@2015 based on data collected from habitat surveys. As emphasised in Chouinard-Thuly et al. [53], the simulations created herein are based on data from real environments. We utilised the habitat of $A$. muricatus from earlier work [27, 36], while the three new habitats were created as close replicates of the actual habitats. Briefly, the habitat models were constructed in accordance with a library of reference images photographed at each site. These included 360 o panoramic photographs to capture the general layout of the habitat, while microhabitat detail was captured by photographing plants and substrates within close range of a known signalling site. The position of plants surrounding the signalling point were noted and spatial layout information was incorporated into each scene.

As Maya does not provide ready-made plant models, major plant species from our study sites, such as spinifex grass and eucalyptus trees, were individually modelled and integrated with precise wind controllers for setting the speed, intensity and frequency of plant movements. The wind controllers are set as a sliding bar to vary in the range $[0,1]$, resulting in increasing movement intensity. Deformation of plant models in response to simulated wind utilised built-in scripts within Maya, which are based on physically accurate equations [54]. By manipulating the numbers on the wind controller, we set the movements of modelled plants to visually match video recordings of actual plant movements in response to moderate wind conditions at each site. Video recordings of plants at each site were taken during periods with natural wind speed close to $0 \mathrm{~m} / \mathrm{s}$ and plant movements were generated using a leaf 
blower positioned 2 and $3 \mathrm{~m}$ from the plant, with wind speeds measured at the plant of 2.5 and $4 \mathrm{~m} / \mathrm{s}$ respectively. The camera was positioned $2 \mathrm{~m}$ from the base of the target plant and angled perpendicular to the direction of the wind emanating from the leaf blower.

\section{D lizard models and display sequences}

Detailed steps on how 3D models of lizards were created are described elsewhere [27]. Lizard display sequences were created using video recordings of each species performing movement-based displays in nature. As described above, the displays of these four species are quite distinct. The temporal structure of the display is presented in Fig. 1, computed as the extent of body movement over time from motion analysis (see below). The head-bob display of $C$. fordigenerates the least movement (Fig. 1b), although relatively continuous throughout the sequence. In contrast, the display of $A$. muricatus is characterised by intermittent movement (Fig. 1a), beginning with small movements at the tip of the tail, then utilising the whole tail before the push-up component of the display. The temporal structure of both $G$. longirostris and $C$. decresii involve relatively constant movement once the display begins (Fig. 1C, d respectively). The display of $C$. decresii involves a gradual increase in the amount of body movement before it ends, while G. longirostris incorporates several rapid changes in body movement (rapid increase and decrease). All lizard and habitat models were built in proportion and to scale.

\section{Scene composition and analysis}

The models of all lizard species were placed into the habitat of all species (Fig. 2a,b; Figure S4). For a given habitat, the lighting conditions, location and orientation of the lizard in the scene and the position of the camera were matched for all lizard species. We used a single directional light to illuminate the entire scene with a uniform lighting effect. Lizard models were positioned in a given habitat according to where the local species would typically be found and orientated side-on to the camera. The animated signalling sequences of all lizard displays started at the same frame in the animation timeline, and were similar in duration ( $6.4 \mathrm{~s}$ or 160 frames, with approximately 5 to 10 frames differences between the set). All sequences were $8 \mathrm{~s}$ duration and exported as images at $25 \mathrm{fps}$ and $720 \times 576$ resolution. We manipulated wind conditions by systematically increasing the extent of plant motion at all sites by manually adjusting plant controllers, confirming incremental (but non-linear) increases in noise using computational motion analysis as described in [36]. We repeated this to achieve 10 versions of each scene varying in environmental noise conditions. All four species were placed in the habitats of each species under all 10 noise conditions resulting in a final set of 160 sequences. For each species-habitat combination, we also created a baseline sequence featuring only lizard movements that is required for our analytical approach.

Our analytical approach to determining salience of lizard displays was the same as previous work [27, 36]. Briefly, animation sequences featuring plant movements were analysed frame by frame using saliency analysis to generate a relative salience score in the range $[0,1]$ for all areas of the frame (Fig. 2ce). The scores of each sequence were then multiplied by its relevant binary mask, which featured only the lizard movement, to produce a relative salience score for the lizard movements in the environmental context imposed. This was repeated for all frames in the sequence to generate the relative salience over 
time (Fig. 2f). As in our earlier work, we then computed the proportion of the sequence that exceeded a given threshold as a function of changing receiver thresholds (1000 values between 0 and 1 varied at increments of 0.01 ; Fig. $2 \mathrm{~g}$ ). A single vector of values is obtained for each species in each wind condition of each habitat.

\section{Data analysis}

Our objective was to compare and contrast the performance of lizard displays in different habitats under varying environmental conditions. We have simulated a single exemplar of each species and habitat to achieve this, so inferential statistics are not appropriate. Nevertheless, our simulations provide novel and informative data that we have considered quantitatively. We began by running non-metric multidimensional scaling (MDS) analysis, and used the metaMDS function from the Vegan package [55] in the R Statistical Environment [57] to quantify similarities/dissimilarities in the resultant vectors for each combination of species, habitat and wind condition (160 vectors). All data were considered together; however, to facilitate visual inspection of results, we group the outcome by wind condition and present the same results twice, labelled by the signalling species and then by habitat. Our second approach was to consider performance not as a single vector, but after segmenting individual datapoints (Fig. $2 \mathrm{~g}$ inset) and grouping into three receiver threshold categories: low (0-0.3), moderate (0.3-0.8), high (0.8-1.0). We first confirmed the detrimental effect of wind for $A$. muricatus in its own habitat, before focusing on moderate receiver thresholds to compare species in all habitats during moderate and strong wind conditions.

\section{Ethical note}

This study was conducted following ASAB/ABS's Guidelines for the treatment of animals in behavioural research and teaching. Fieldwork undertaken to film lizard displays was completed with approval from La Trobe University's Animal Ethics Committee (AEC 16-59) and under permits from Australian state government authorities: DEWLP Wildlife Research Permit No. 10008006 (Victoria), DEWNR Wildlife Permit No. U26541 (South Australia) and Parks and Wildlife Commission Permit No. 59056 (Northern Territory).

\section{Results}

The effectiveness of displays by four Australian agamid lizard species were assessed in their own habitats and the habitats of each of the other species under different wind conditions. In each case, we obtained the proportion of the sequence that exceeded a specified threshold for response as a function of varying threshold values. Figure 3 provides the result of non-metric MDS analysis comparing similarities/dissimilarities between all 160 resultant vectors. The $X Y$ coordinate data are presented separately for calm (levels 1-3), moderate (levels 4-7) and strong (levels 8-10) wind conditions. With datapoints labelled by the species displaying (Fig. 3a), we observe some grouping by species, though these appear in clusters of a few points (i.e., a single habitat) rather than as a single species cluster that would imply similar performance across all habitats and conditions. This is consistent with the notion that performance of a given display is dependent on location. Another observation we can make here is that the performance of three species (A. muricatus, G. longirostris and $C$. decresii) are comparable in 
certain habitats (Fig. 3c, blue lines), while one species, $C$. fordi, is consistently on the outer and performs relatively poorly (Fig. 3c, red lines). In Fig. 3b we label data by the habitat in which displays are performed. What is most striking here is that displays by all species within the habitat of $A$. muricatus cluster together, and this gets tighter as wind conditions worsen. Performance in this habitat is strong to begin with, but declines as receivers become more discriminating (Fig. 3c, black lines), suggesting that the task of distinguishing signal from noise has become more difficult.

We next considered the data as individual performance scores grouped into low, moderate and high receiver threshold categories. Figure 4 provides the outcome for $A$. muricatus in its own habitat. When receivers are non-discriminating, display performance is strong even as wind conditions increase. However, the masking effect of wind becomes apparent when receivers start to be more selective in what will attract attention. Given highly selective receivers are predicted to rarely detect a signal in strong wind conditions, we chose to focus on moderate receiver response thresholds to compare species across all habitats. The performance of each species' display in each habitat is shown in Fig. 5 for moderate and strong wind conditions (Fig. 5). These data confirm our earlier observations that the habitat of $A$. muricatus is the most detrimental to display performance, while the display of $C$. fordi is consistently the worst performing. We also see that stronger wind reduces performance across all sites (scores reduced in Fig. 5b compared with Fig. 5a), although to varying degrees (greater spread in data in strong winds).

\section{Discussion}

Technical difficulties in quantifying environmental conditions and movement-based displays in a systematic way has made examination of movement-based signals a challenging task. Legislative restrictions have made in situ cross-habitat comparisons improbable. Recent studies have attempted to contrast multiple signals in multiple habitats by quantifying signal and noise separately and then contrasting resultant motion speed data [33-34]. For the first time, sophisticated 3D animations gave us the opportunity to closely examine movement-based signals in multiple habitats. Specifically, we were able to quantify how varying environmental conditions, receiver behaviour and differences in display and microhabitat structures effected signal efficacy. Using four Australian agamid lizard species, we compared and contrasted how species-specific movement-based signals would perform in structurally distinct environments. Our simulations suggested that signal efficacy varies as a function of receiver response thresholds, where at medium to high thresholds, the changes within and between species became clear. In line with our predictions, all signals performed the worst in $A$. muricatus habitat, the performance of $G$. longirostris and $C$. decresii were very similar and the display of $C$. fordi was the worst across all habitats. However, contrary to our prediction, the performance of $A$. muricatus was not more effective than $G$. longirostris and $C$. decresii, so the notion that signals expected to be adapted to a noisier environment will perform better than the ones adapted to less noisy environments was not supported.

The poor performance of the display by $C$. fordi relative to other species was anticipated and might be attributable to differences in signal function. Unlike the other three species in the study, $C$. fordi are not territorial and their displays are not considered aggressive responses to intruders. It has been suggested 
that the likely function of the simple head bob display is for species recognition [46]. Displays are performed at close range when lizards encounter other lizards, so the necessity to distinguish itself from surrounding plant motion is removed. The other three species in this study are all assumed to be territorial species $[42,52,57]$, responding to intruders at greater distances and when the intruder is possibly unaware of the presence of the territory owner. In these circumstances, reliable detection and processing of territorial displays are essential for establishing social dominance [58-59].

The potential masking effect for movement-based signals is dependent on prevailing wind and the distribution of plants in the environment [32]. Complex image motion backgrounds are expected in dense vegetation, which would likely require animals to generate certain types of movements for effective communication [3]. The habitat of $A$. muricatus is densely vegetated with tall grasses and small shrubs (Figure S1a). The plants are often positioned close to the signalling animal, with short signaller-plant distances predicted to reduce detectability of signals [60]. In comparison, the habitats of G. longirostris and $C$. decresii feature sparsely vegetated environments, with plants typically separated from the signalling animals. However, the $C$. decresii habitat we modelled herein was based on a recently burnt area which would feature a reduced level of motion noise in the environment, this information could change for $C$. decresii as the vegetation recovers from the fire over the next few years. The signaller-plant distances in the habitat of $C$. fordi were also very close, but in this case the dominant plant species, spinifex grass (Triodia sp.), does not generate considerable movement in response to wind at the base of the plant where the lizards were positioned. As such, the potential masking effect of plant environments is attributable to the interaction between wind conditions, the spatial position of plants in the environment and the structure of the plant itself that will dictate how it responds to wind.

Habitats with common structural properties, regardless of geographic location, are predicted to result in signals with similar structural characteristics [61-62]. Both $G$. longirostris and $C$. decresii inhabit sparsely vegetated rocky outcrops, and the similarities in the structure of their signals and relative signal performance in noise could be attributed to structural similarities in the environments they inhabit. Interestingly, the performance of their displays was just as effective as the signal of $A$. muricatus in the habitat of $A$. muricatus suggesting different movements might be equally effective. However, it is in the other habitats that we start to see subtle interactions between display and habitat structure. The displays of $G$. longirostris perform best in two of these habitats, while $C$. decresii displays perform better in their own habitat. Ramos and Peters [48] found no evidence for broad scale habitat characteristics influencing signal use in Australian dragons, but it is intriguing to consider the possibility that the use of different motor patterns is partially driven by fine-scale habitat characteristics that influence the motion noise environment. Both $G$. longirostris and $C$. decresii signals involve almost continuous movement featuring whole body push-ups/forward thrusts. In contrast, the push-up component of the display of $A$. muricatus is brief and follows an occasionally lengthy period of tail flicking [14]. Tail-flicks represent the introductory component to the rest of the display, reportedly to capture the attention of a receiver $[14,43]$. It is also this component of the signal that exhibits plasticity in response to changing environmental conditions [14], whereby the duration of tail flicking is lengthened in noisy conditions and the movement becomes more intermittent. Tail-flicks are observed in $C$. decresii, although it is not a core component of 
the display [35], and there are no reports for tail flicking by G. longirostris [48]. Further research is needed to determine whether either of these species possess a component of their display that exhibits the kind of plasticity needed for variable environments. Alternatively, perhaps the environments that they inhabit have not selected for such plasticity as the rocky gorges provide a clear background against which the signal can be reliably detected regardless of plant movement speeds.

This study has added to a growing literature showing that habitat characteristics and the associated noise landscape has a significant evolutionary effect on signal design and likely promotes changes in signalling strategies under certain conditions [14, 63-64]. How signals function in social interactions is another important consideration for driving variation in signal structure [46, 58,65-66]. Cross-habitat examination of signal performance has been beneficial for other communication systems $[19,20,21,23]$, but the present study is one of the first comparable studies for movement-based signals. Our innovative approach allowed for examination of different movement-based signals embedded in noise across different habitats. We acknowledge that the results herein are generated from a single representative display per species, in a single representative habitat per species. Nevertheless, our method and analytical approach has provided quantitative data showing that the effectiveness of movement-based animal signals will be affected by the motion noise environment, and might indeed direct signalling behaviour in much the same way that it has for other modes of signalling.

\section{Declarations}

\section{Acknowledgements}

We are grateful to Bhagya Herath, Yin Qi and Jose Ramos for assistance with fieldwork to film lizard displays and document habitat characteristics. In addition, we thank Warwick Laird and Chandara Ung for assistance with preliminary animations.

\section{Funding}

This work was supported by an Australian Research Council Discovery Project grant to RAP and TC (DP170102370).

\section{Author Contributions}

XB and RAP designed the study and conducted fieldwork

$\mathrm{XB}, \mathrm{AP}$ and $\mathrm{TC}$ created the $3 \mathrm{D}$ animations

$\mathrm{XB}$ and RAP analysed the rendered animations and wrote the first draft of the manuscript.

All authors provided comments on earlier drafts and finalised the manuscript

\section{References}


1. Endler, J. A. 1992. Signals, signal conditions, and the direction of evolution. Nat. 139, S125-S153.

2. Endler, J. A. 1993. Some general comments on the evolution and design of animal communication systems. Trans. R. Soc. Lond. B. Biol. Sci. 340, 215-225.

3. Fleishman, L. J. 1992. The influence of the sensory system and the environment on motion patterns in the visual displays of anoline lizards and other vertebrates. Nat. 139, S36-S61.

4. Lythgoe, J. N. 1979. The Ecology of vision. Oxford, Oxford University Press.

5. Bradbury, J. W. and Vehrencamp, S. L. 1998. Principles of animal communication $\left(2^{\text {nd }}\right)$. Sinauer Associates.

6. Morton, E. S. 1975. Ecological Sources of Selection on Avian Sounds. Nat. 109, 17-34.

7. Endler, J. A. 1990. On the measurement and classification of colour in studies of animal colour patterns. J. Linn. Soc. Lond. 41, 315-352.

8. Wiley, R. H. and Richards, D. G. 1983. Adaptations for acoustic communication in birds: sound transmission and signal detection. In: Ecology and Evolution of Acoustic Communication in Birds. Kroodsma D. E. and Miller E. H. (eds). New York, Academic Press, pp 131-181.

9. Bernard, G. D. and Remington, C. L. 1991. Color vision in Lycaena butterflies: spectral tuning of receptor arrays in relation to behavioral ecology. Natl. Acad. Sci. USA. 88, 2783-2787.

10. Peters, R. A., Clifford, C. W. G. and Evans, C. S. 2002. Measuring the structure of dynamic visual signals. Behav. 64, 131-146.

11. Narins, P. M. 1990. Seismic communication in anuran amphibians. Bioscience 40, 268-274.

12. Fleishman, L. and Persons, M. 2001. The influence of stimulus and background colour on signal visibility in the lizard Anolis cristatellus. Exp. Biol. 204, 1559-1575.

13. Brumm, H. and Slabbekoorn, H. 2005. Acoustic communication in noise. Study Behav. 35, 151-209.

14. Peters, R. A., Hemmi, J. M. and Zeil, J. 2007. Signaling against the wind: modifying motion-signal structure in response to increased noise. Biol. 17, 1231-1234.

15. Ord, T. J. and Stamps, J. A. (2008). Alert signals enhance animal communication in "noisy" environments. Natl. Acad. Sci. USA. 105, 18830-18835.

16. Komers, P. E. 1997. Behavioural plasticity in variable environments. J. Zool. 75, 161-169.

17. Ord, T.J., Charles, G. K., Palmer, M. and Stamps, J. A. 2015. Plasticity in social communication and its implications for the colonization of novel habitats. Ecol. 27b 341-351.

18. Marten, K. and Marler, P. 1977. Sound transmission and its significance for animal vocalization. Ecol. Sociobiol. 2, 271-290.

19. Ryan, M. J., Cocroft, R. B. and Wilczynski, W. 1990. The role of environmental selection in intraspecific divergence of mate recognition signals in the cricket frog, Acris Crepitans. Evolution 44, 1869-1872

20. Leal, M. and Fleishman, L. J. 2004. Differences in visual signal design and detectability between allopatric populations of Anolis Am. Nat. 163, 26-39. 
21. McNett, G. D. and Cocroft, R. B. 2008. Host shifts favor vibrational signal divergence in Enchenopa binotata Behav. Ecol. 19, 650-656.

22. Ferguson, G. W. 1971. Variation and evolution of the push-up displays of the side-blotched lizard genus Uta (Iguanidae). Zool. 20, 79-101.

23. Martins, E. P., Bissell, A. N. and Morgan, K. K. 1998. Population differences in a lizard communicative display: evidence for rapid change in structure and function. Behav. 56, 1113-1119.

24. Martins, E. P. and Lamont, J. 1998. Estimating ancestral states of a communicative display: a comparative study of Cyclurarock iguanas. Behav. 55, 1685-1706.

25. Bloch, N. and Irschick, D. 2006. An analysis of inter-population divergence in visual display behavior of the green anole lizard (Anolis carolinensis). Ethology 112, 370-378.

26. Barquero, M. D., Peters, R. and Whiting, M. 2015. Geographic variation in aggressive signalling behaviour of the Jacky dragon. Ecol. Sociobiol. 69, 1501-1510.

27. Bian, X., Chandler, T., Laird, W., Pinilla, A. and Peters, R. 2018. Integrating evolutionary biology with digital arts to quantify ecological constraints on vision-based behaviour. Methods Ecol. Evol. 9, 544559.

28. Fleishman, L. J. 1986. Motion detection in the presence and absence of background motion in an Anolis J. Comp. Physiol. A 159, 711-720.

29. Fleishman, L. J. 1988. Sensory and environmental influences on display form in Anolis auratus, a grass anole from Panama. Ecol. and Sociobiol. 22, 309-316.

30. Eckert, M. P. and Zeil, J. 2001. Towards an ecology of motion vision. In: Motion Vision. Zanker J.M and Zeil J. (eds). Springer, pp 333-369.

31. Peters, R. A. and Evans, C. S. 2003. Design of the Jacky dragon visual display: signal and noise characteristics in a complex moving environment. Comp. Physiol. A 189, 447-459.

32. Peters, R. A. 2013. Noise in visual communication: motion from wind-blown plants. In: Animal Communication and Noise. Animal Signals and Communication Brumm H. (eds) Springer, Berlin Heidelberg, pp. 311-330.

33. Ramos, J. A. and Peters, R. A. 2017. Motion-based signaling in sympatric species of Australian agamid lizards. Comp. Physiol. A 203, 661-671.

34. Ramos, J. A. and Peters, R. A. 2017. Habitat-dependent variation in motion signal structure between allopatric populations of lizards. Behav. 126, 69-78.

35. Ramos, J. A. and Peters, R. A. 2017. Quantifying ecological constraints on motion signaling. Ecol. Evol. 5, 9.

36. Bian, X., Chandler, T., Pinilla, A. and Peters, R. 2019. Now you see me, now you don't: environmental conditions, signaler behavior, and receiver response thresholds interact to determine the efficacy of a movement-based animal signal. Ecol. Evol. 7, 130.

37. Heatwole, H. and Firth, B. T. 1982. Voluntary maximum temperature of the jacky lizard, Amphibolurus muricatus. Copeia, 1982, 824-829. 
38. Harlow, P. S. and Taylor, J. E. 2000. Reproductive ecology of the jacky dragon (Amphibolurus muricatus): an agamid lizard with temperature-dependent sex determination. Ecol. 25, 640-652.

39. Ord, T. J. and Evans, C. S. 2003. Display rate and opponent assessment in the Jacky dragon (Amphibolurus muricatus): an experimental analysis. Behaviour 140, 1495-1508.

40. Warner, D. A. and Shine, R. 2010. Interactions among thermal parameters determine offspring sex under temperature-dependent sex determination. R. Soc. Lond. B. Biol. Sci. 278, 256-265.

41. Carpenter, C. C., Badham, J. A. and Kimble, B. 1970. Behavior patterns of three species of Amphibolurus (Agamidae). Copeia, 1970, 497-505.

42. Peters, R. A. and Ord, T. J. 2003. Display response of the Jacky Dragon, Amphibolurus muricatus (Lacertilia : Agamidae), to intruders: a semi-Markovian process. Ecol. 28, 499-506.

43. Peters, R. A. and Evans, C. S. 2003. Introductory tail-flick of the Jacky dragon visual display: signal efficacy depends upon duration. Exp. Biol. 206, 4293-4307.

44. Wilson, S. and Swan, G. 2013. A Complete guide to reptiles of Australia (second ed.) Reed New Holland, Sydney.

45. Carpenter, C. C. 1962. A comparison of the patterns of display of Urosaurus, Uta, and Streptosaurus. Herpetologica 18, 145-152.

46. Cogger, H. 1978. Reproductive cycles, fat body cycles and socio-sexual behaviour in the mallee dragon, Amphibolurus fordi (Lacertilia: Agamidae). J. Zool. 26, 653-672.

47. Garcia, J. E., Rohr, D. and Dyer, A. G. 2013. Trade-off between camouflage and sexual dimorphism revealed by UV digital imaging: the case of Australian Mallee dragons (Ctenophorus fordi). Exp. Biol. 216, 4290-4298.

48. Ramos, J. A. and Peters, R. A. 2016. Dragon wars: movement-based signalling by Australian agamid lizards in relation to species ecology. Ecol. 41, 302-315.

49. Gibbons, J. R. H. 1977. Comparative ecology and behaviour of lizards of the Amphibolurus decresii species complex. PhD dissertation, University of Adelaide, Adelaide, South Australia

50. McLean, C. A., Moussalli, A., Sass, S. and Stuart-Fox, D. 2013. Taxonomic assessment of the Ctenophorus decresii complex (Reptilia: Agamidae) reveals a new species of dragon lizard from western New South Wales. Aust. Mus. 65, 51-63.

51. Osborne, L. 2005. Information content of male agonistic displays in the territorial tawny dragon (Ctenophorus decresii). Ethol. 23, 189-197.

52. Gibbons, J. R. 1979. The hind leg pushup display of the Amphibolurus decresii species complex (Lacertilia: Agamidae). Copeia, 1979, 29-40.

53. Chouinard-Thuly, L., Gierszewski, S., Rosenthal, G. G., Reader, S. M., Rieucau, G., Woo, K. L., Gerlai, R., Tedore, C., Ingley, S. J. and Stowers, J. R. 2016. Technical and conceptual considerations for using animated stimuli in studies of animal behavior. Zool. 63, 5-19

54. Akagi, Y. and Kitajima, K. (2006). Computer animation of swaying trees based on physical simulation. Graph. 30, 529-539. 
55. Oksanen, J., Blanchet, F. G., Friendly, M., Kindt, R., Legendre, P., McGlinn, D., Minchin, P. R., O'Hara, R. B., Simpson, G. L., Solymos, P., Stevens, M. H. H., Szoecs, E. and Wagner, H. 2019. Vegan: Community Ecology Package. $\mathrm{R}$ package version 2.5-4. https://CRAN.R-project.org/package=vegan

56. R Core Team. 2018. R: A language and environment for statistical computing. R Foundation for Statistical Computing, Vienna, Austria. URL https://www.R-project.org/.

57. Blamires, S. 1998. Circumduction and head bobbing in the agamid lizard Lophognathus temporalis. Herpetofauna 28, 51-52.

58. Carpenter, C. C. 1967. Aggression and social structure in iguanid lizards. In: Lizard ecology. A symposium. Milstead W. W. (eds.). University of Missouri Press Columbia.

59. Carpenter, C. 1978. Ritualistic social behaviors in lizards. In: Behavior and Neurology of Lizards, an interdisciplinary colloquium. Rockville, Maryland: National Institute of Mental Health, Rockville. Pp. 253-267.

60. Peters, R. A., Hemmi, J. and Zeil, J. 2008. Image motion environments: background noise for movement-based animal signals. Comp. Physiol. A 194, 441-456.

61. Hunter, M. L. and Krebs, J. R. 1979. Geographical variation in the song of the great tit (Parus major) in relation to ecological factors. Anim. Ecol 48, 759-785.

62. Harmon, L. J., Kolbe, J. J., Cheverud, J. M. and Losos, J. B. 2005. Convergence and the multidimensional niche. Evolution 59, 409-421.

63. Fleishman, L. J. 1988. Sensory influences on physical design of a visual display. Behav. 36, 14201424.

64. Ord, T. J., Peters, R. A., Clucas, B. and Stamps, J. A. 2007. Lizards speed up visual displays in noisy motion habitats. R. Soc. Lond. B. Biol. Sci. 274, 1057-1062.

65. Hasson, O. 1991. Pursuit-deterrent signals: communication between prey and predator. Trends Ecol. Evol. 6, 325-329.

66. Hebets, E. A. and Uetz, G. W. 1999. Female responses to isolated signals from multimodal male courtship displays in the wolf spider genus Schizocosa (Araneae: Lycosidae). Behav. 57, 865-872.

\section{Figures}



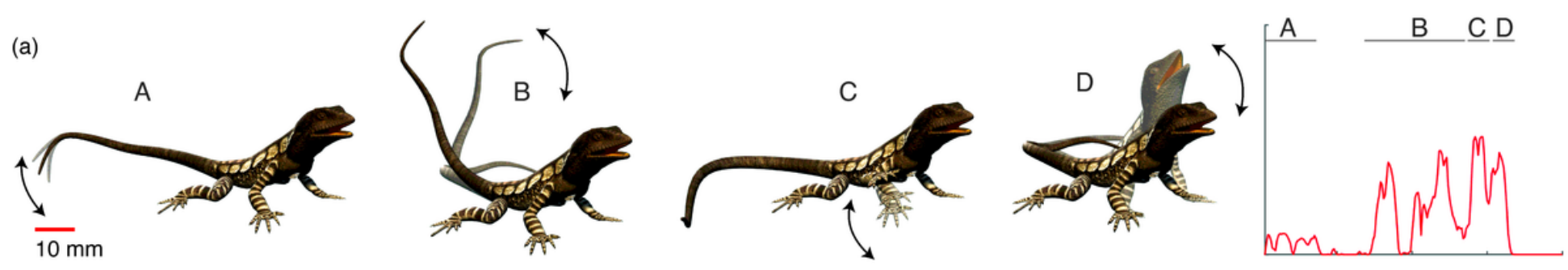

(b)
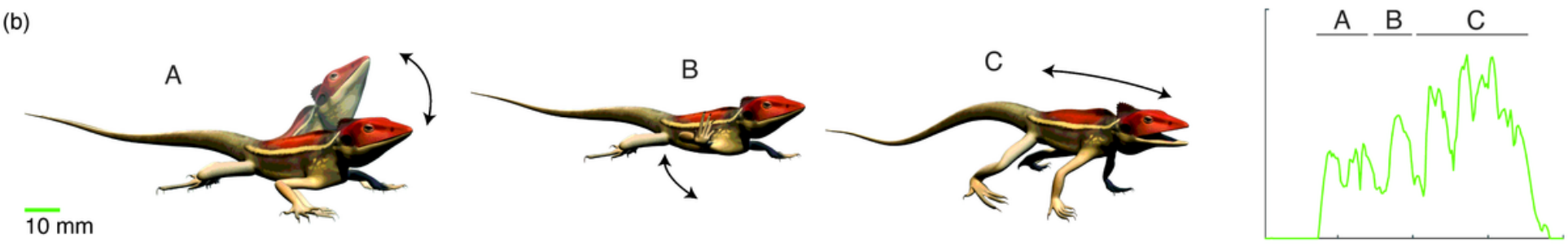

(c)

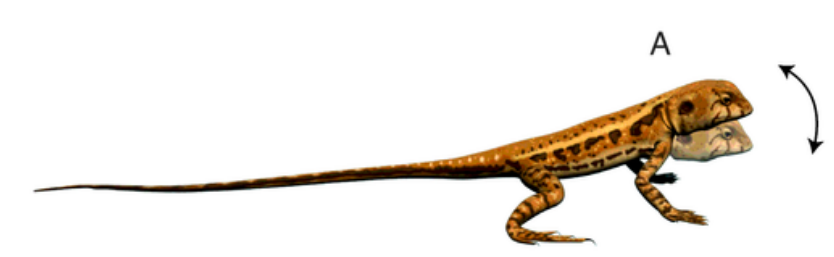

$\overline{10 \mathrm{~mm}}$

(d)

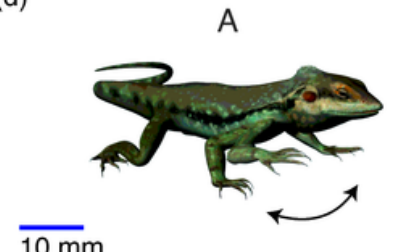

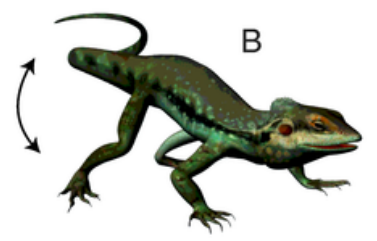

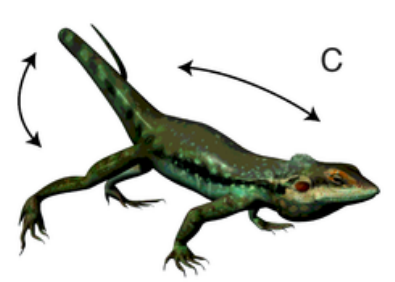

A
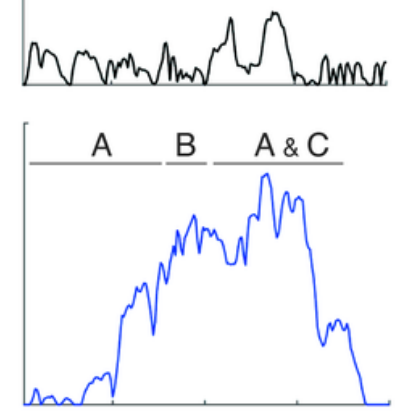

\section{Figure 1}

Images depicting the display sequences of the four lizard species used in the present study (left) accompanied by the respective temporal structure profile graph (right; see text for details). In each image set, black arrows indicate the direction of movement. Motions such as head- bobs, limb waves and tailflicks are usually repeated for several cycles. (a) The display sequence of A. muricatus starts with tailflicks $(A, B)$, followed by limb waves (C) and finishes with a whole body movement centred on a push-up (D). The temporal structure graph indicates a relatively intermittent movement throughout the sequence. (b) The display sequence of $G$. longirostris comprises a series of head bobbing (A) and limb waving (B), the final push up is followed by a forward body thrust/rise (C). The temporal structure graph indicates relatively constant movement but with several rapid changes in movements throughout the whole sequence. (c) The display of $C$, fordi is comparatively simple, and consists of rapid movement of the head up and down (head- bobbing, A). The temporal structure graph indicates a relatively continuous movement throughout the sequence. (d) The display sequence of $C$. decresii comprises with several limb waves (A) and a distinct raising of the tail with a coil at the distal end of the tail (B), it finishes with a forward body thrust with tail coiled (C). The temporal structure graph indicates relatively constant 
movement, featuring a gradual increase in the amount of body movement. Scale bars are provided for each species.

(a)
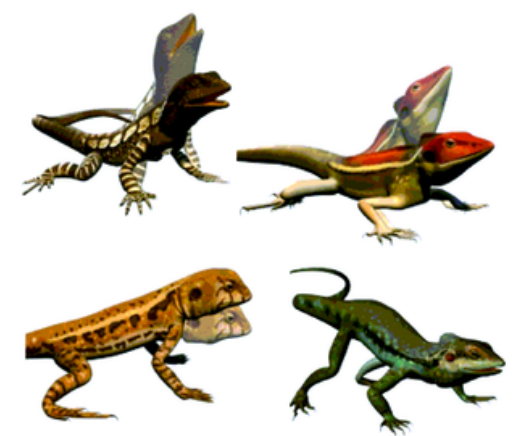

(b)
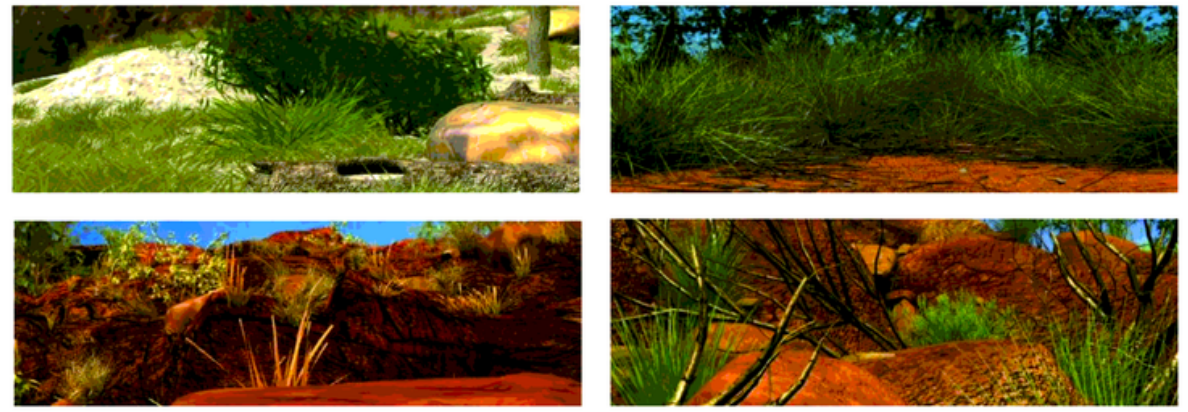

(c)

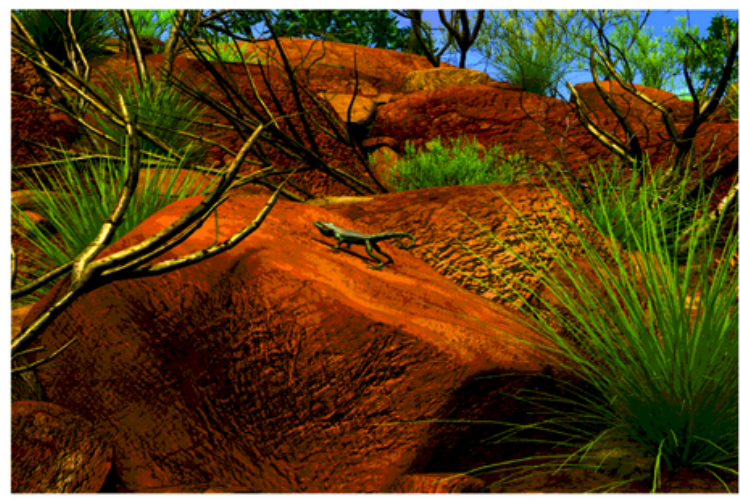

(d)

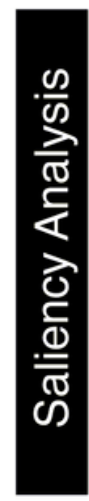

(e)

(g)

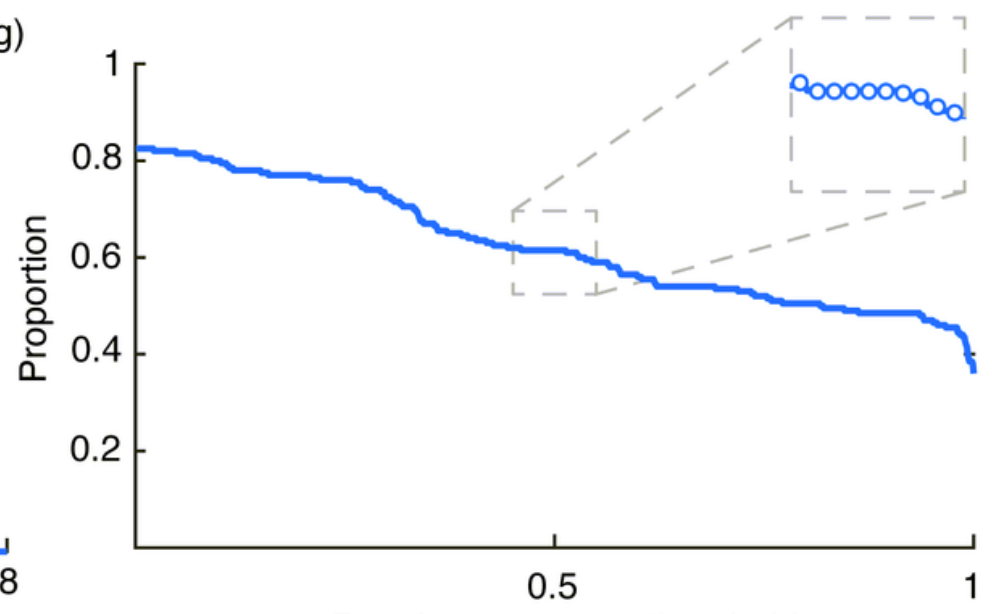

Receiver response threshold
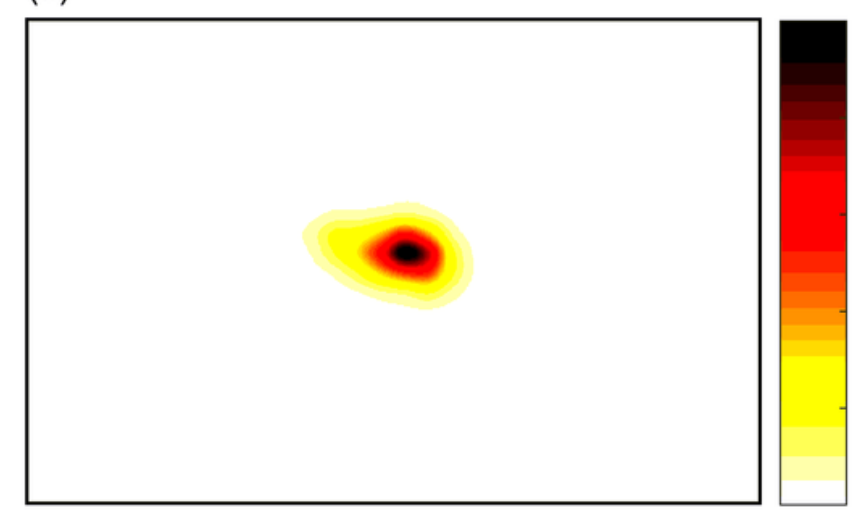

(f)

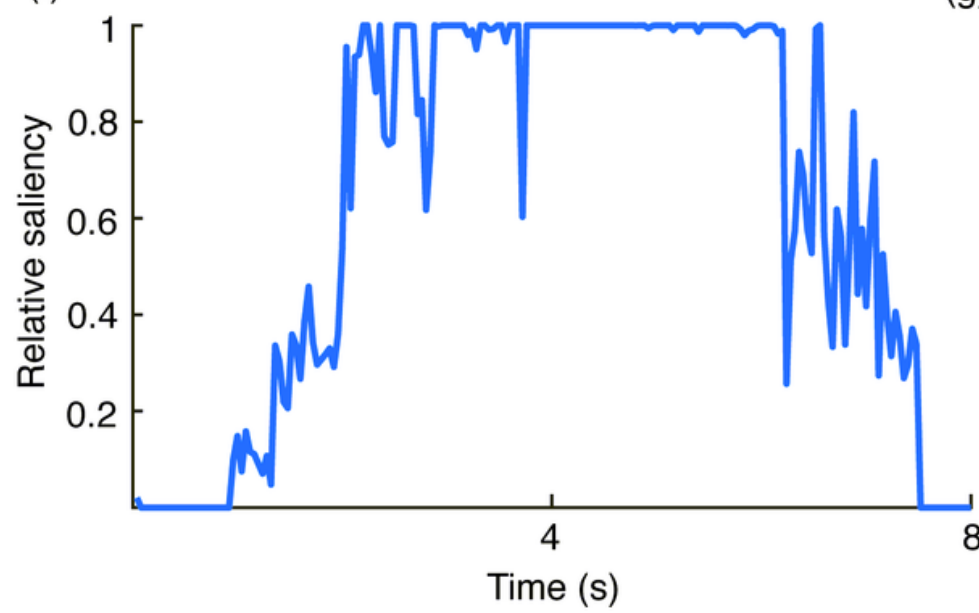

\section{Figure 2}

To examine the relative efficacy of movement-based animal signals we created 3D animations of the displays of four Australian dragon lizards (a) and their respective habitats (b). Each species was inserted into each habitat (c) under varying environmental conditions. We used saliency analysis (d) to compute saliency scores for all parts of the scene (e). The maximum saliency score in the area of the scene featuring the lizard is identified for all frames in the animation sequence ( $f$ ). We assumed the level of salience required to capture attention will vary within and between receivers and determined the proportion of the sequence that exceeded a given threshold as a function of varying receiver response 
thresholds (g). The performance vector comprises discrete scores at small increments in threshold values (g, inset).

(a)

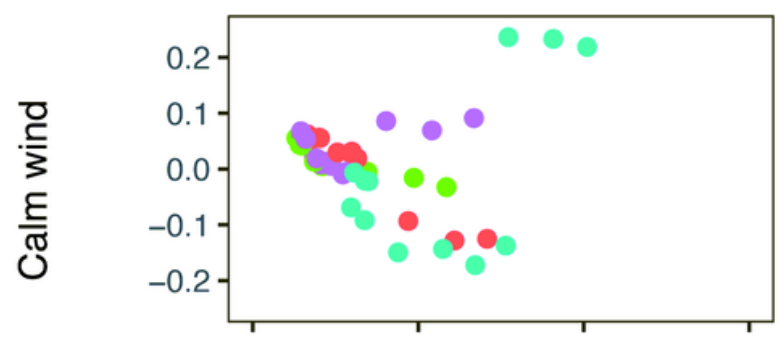

$\frac{1}{3}$
$\frac{0}{4}$
$\frac{0}{\pi}$
$\frac{0}{0}$
$\frac{0}{2}$

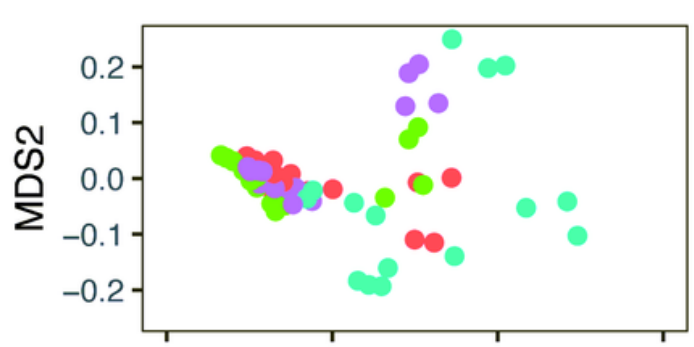

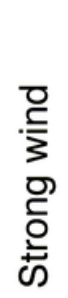

(c)

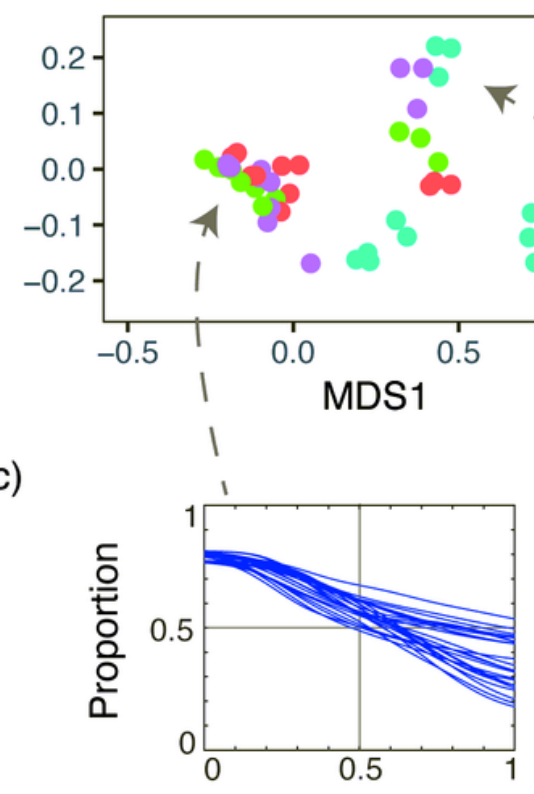

(b)
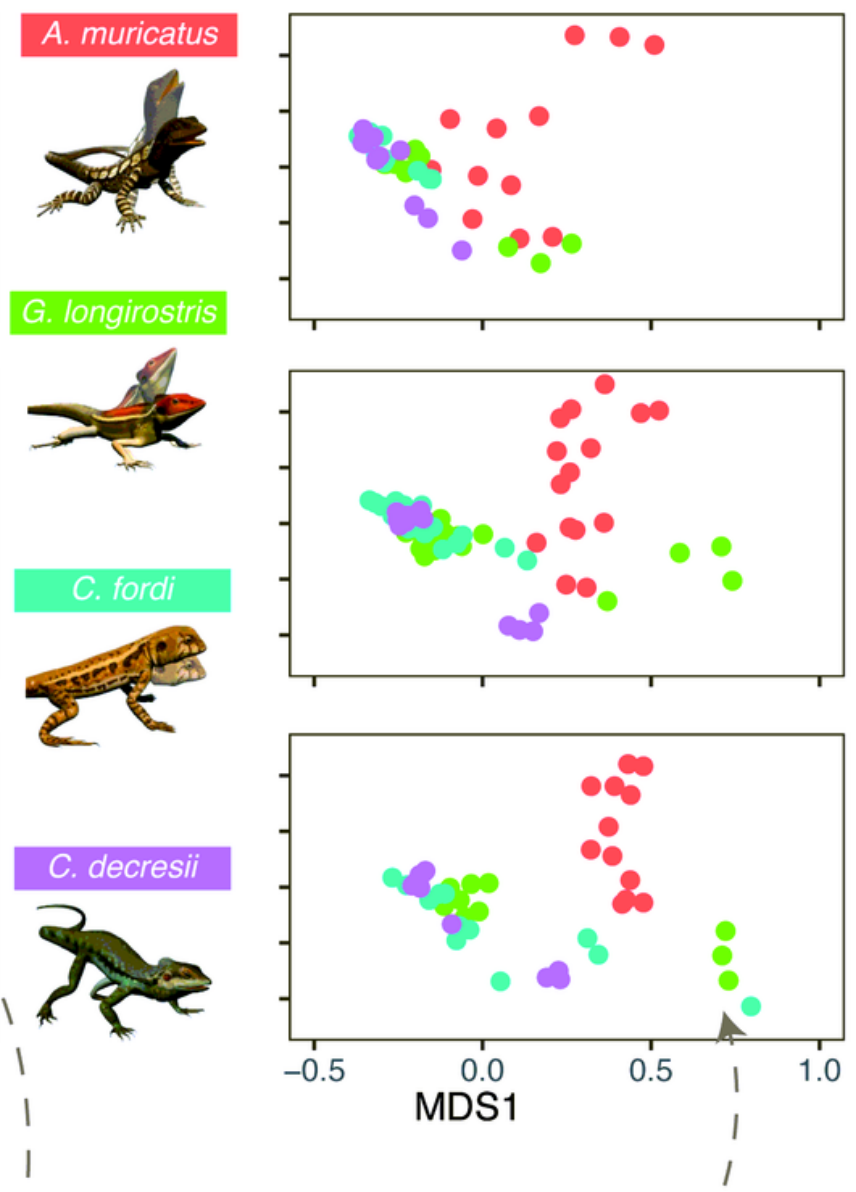

G. Iongirostris
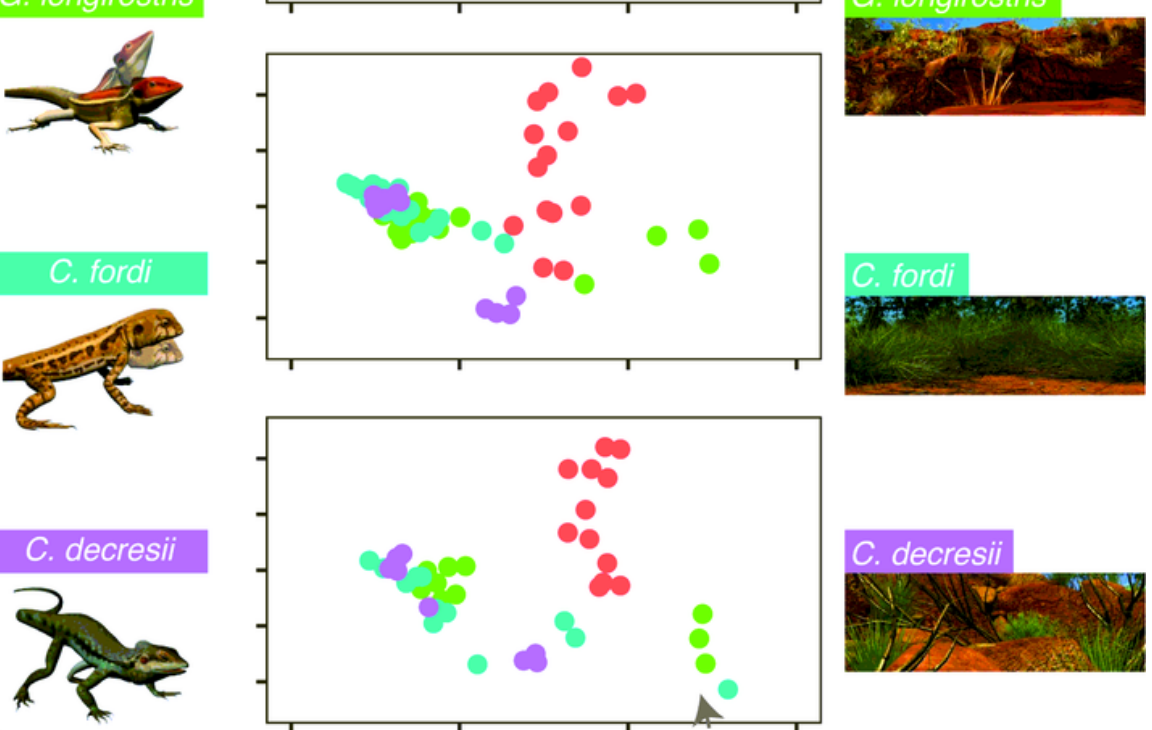

\section{Figure 3}

Results of non-metric multidimensional scaling analysis to compare similarities/dissimilarities between vectors summarising performance of lizard displays in multiple habitats under varying wind conditions. The results are shown twice, labelling data points by the lizard species (a) and by the habitat in which the lizard was placed (b). Separate plots show performance in calm (top row), moderate (middle row) and strong (bottom row) wind conditions. (c) To provide insight into the different clumping within MDS space, individual performance vectors are shown in strong wind for the best performing (blue lines), as well as poorer performing (black and red lines) display species-habitat combinations. 


\section{Receiver Response Threshold}

\section{Low}

$1.00-$

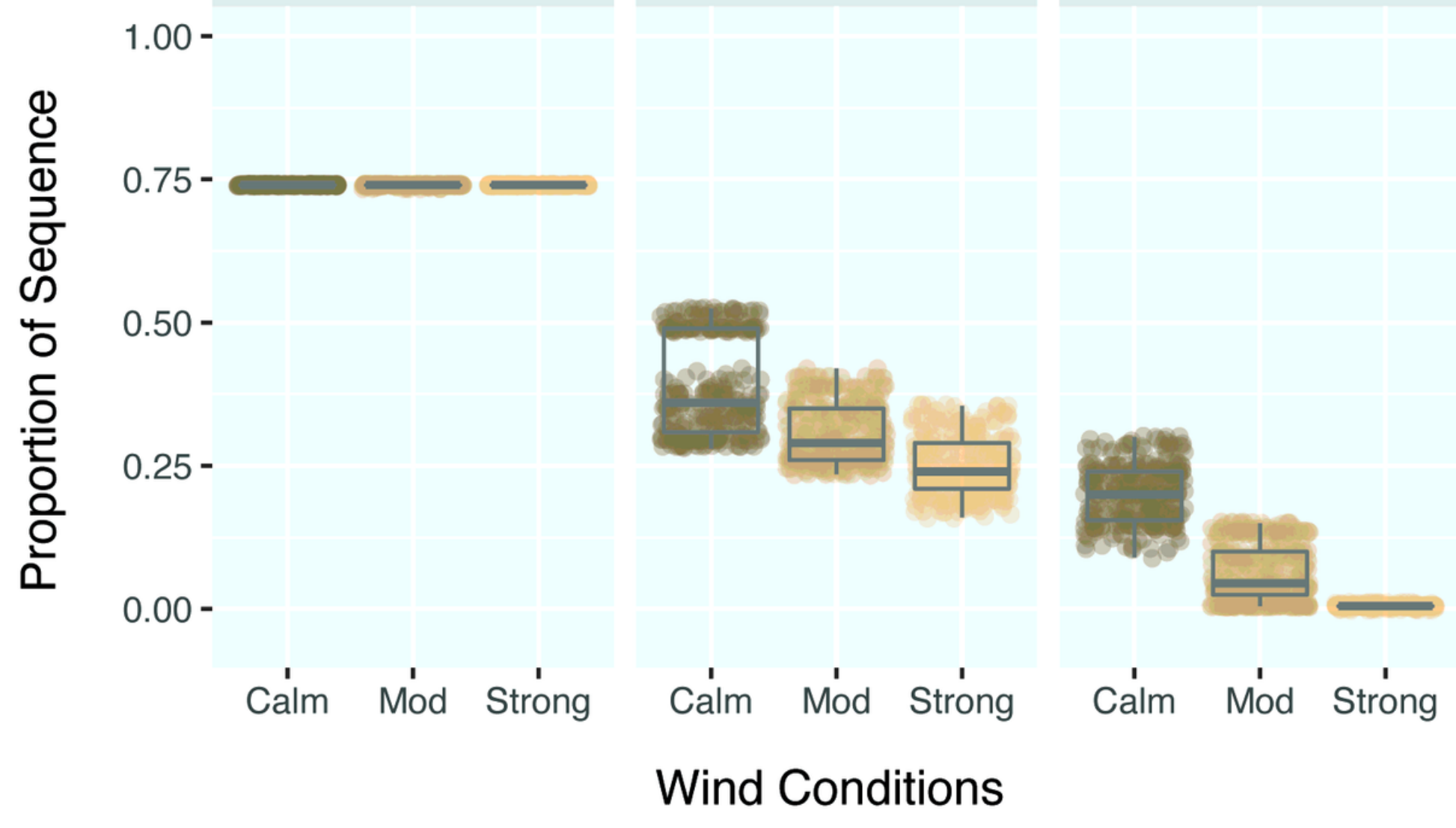

Medium

High

Figure 4

Results of non-metric multidimensional scaling analysis to compare similarities/dissimilarities between vectors summarising performance of lizard displays in multiple habitats under varying wind conditions. The results are shown twice, labelling data points by the lizard species (a) and by the habitat in which the lizard was placed (b). Separate plots show performance in calm (top row), moderate (middle row) and strong (bottom row) wind conditions. (c) To provide insight into the different clumping within MDS space, individual performance vectors are shown in strong wind for the best performing (blue lines), as well as poorer performing (black and red lines) display species-habitat combinations. 


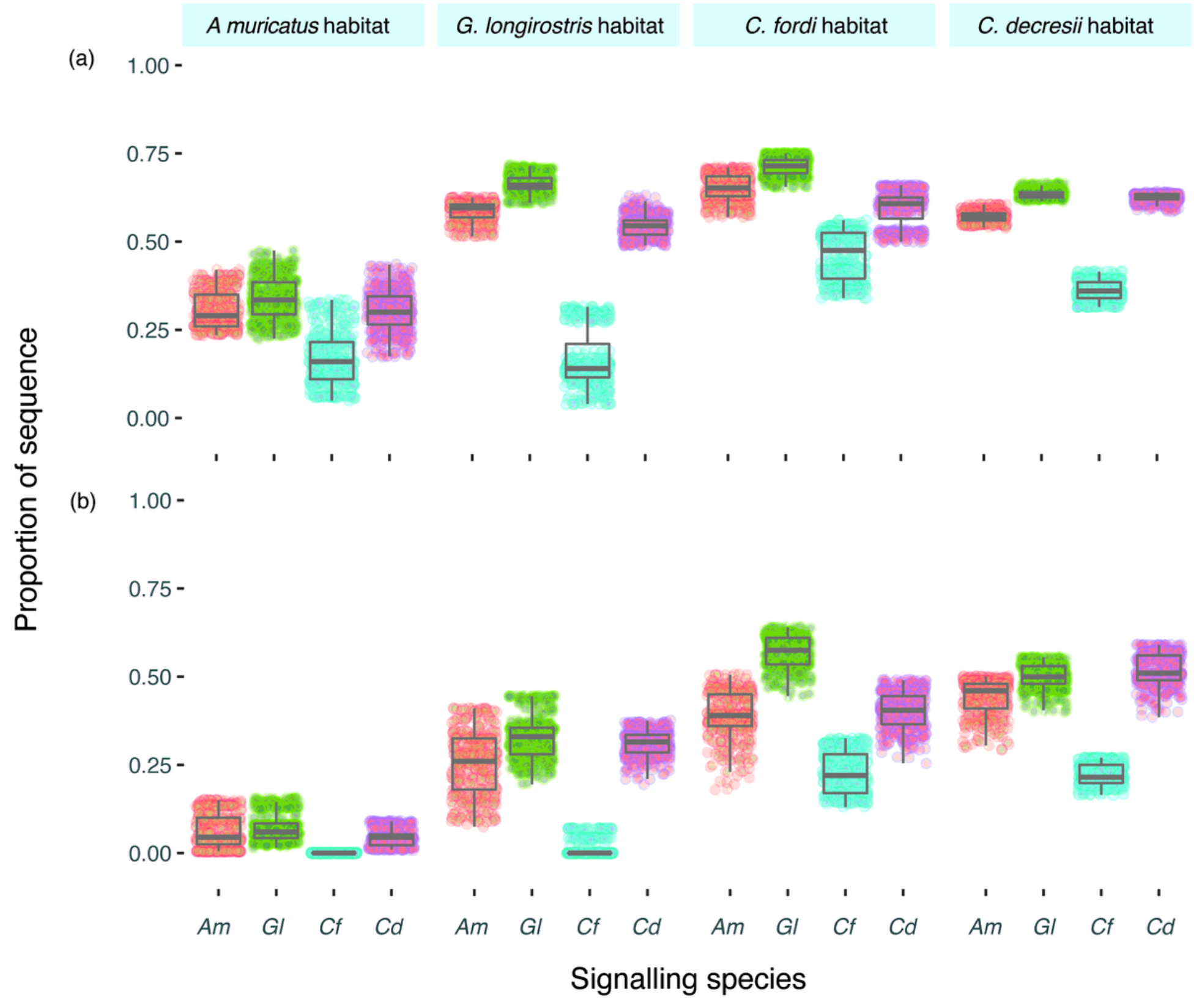

Figure 5

The relative performance of lizard displays under moderate (a) and strong (b) wind conditions with medium receiver response thresholds (0.3-0.8). Separate plots are showing for the habitats in which signalling takes place, with each showing the proportion of the sequence exceeding the threshold shown as a function of signalling species. (Am - A. muricatus; $G L-G$. longirostris; $C f-C$. fordi; $C d-C$. decresii)

\section{Supplementary Files}

This is a list of supplementary files associated with this preprint. Click to download.

- BianPinillaChandlerPetersSupplementaryMaterial.pdf 